\title{
Los marcos de los noticieros televisivos y la segunda pantalla en Twitter y Facebook Escándalo Pacogate en Chile
}

\author{
The Frames of Television News and the Second Screen on Twitter and Facebook: \\ Pacogate Scandal in Chile
}

Francisco Javier Tagle Montt, Juan José Guerrero Cortés y María Paz Vial Cerda*

Resumen: A partir del caso de corrupción Pacogate, que afectó a la policía chilena durante 2017, esta investigación estudia los marcos de los principales noticieros de la televisión chilena y los comentarios en segunda pantalla que se sucedieron en Facebook y Twitter. A través de los encuadres propuestos por Entman (2012), sobre cómo los medios encuadran la corrupción, se postula, como parte del mismo fenómeno mediático, que mientras los noticiarios promovieron con sus marcos una definición y explicación del caso, las opiniones de las redes sociales no sólo fueron polarizadas, sino que también dieron una dimensión moralizante y crítica de lo ocurrido.

Palabras clave: escándalo, encuadres, televisión, redes sociales, Pacogate, second screen.

Abstract: Based on the Pacogate corruption case, which affected the Chilean police during 2017, this research explores, using an exploratory methodology and a mixed methodology, the frameworks of the main Chilean television news and the comments that followed on Facebook and Twitter. Through the frames proposed by Entman (2012) on how the media frame corruption, it is postulated, as part of the same media phenomenon, that while the news programs promoted with their frameworks a definition and explanation of the case, the opinions of social networks not only were polarized, but they also gave a moralizing and critical dimension to what happened.

Keywords: scandal, framing, television, social networks, Pacogate, second screen.

*Francisco Javier Tagle Montt es académico de la Facultad de Comunicación de la Universidad de los Andes, Chile, Monseñor Âlvaro del Portillo 12.455, Las Condes, Santiago de Chile, 7620001. Tel: +56 2618 1000. Correo-e: fjtagle@uandes.cl. orciD: http://orcid.org/0000-0003-1480-1020. Juan José Guerrero Cortés es académico de la Facultad de Comunicación de la Universidad de los Andes, Chile, Monseñor Álvaro del Portillo 12.455, Las Condes, Santiago de Chile, 7620001. Tel: +56 2618 1000. Correo-e: JJGUERRERO@uandes.cl. orCiD: https://orcid.org/0000-0002-7286-3660. María Paz Vial Cerda, Universidad de los Andes, San Carlos de Apoquindo 2200, Las Condes, Santiago de Chile, 7620001. Correo-e: mpvial1@miuandes.cl. orCID: https://orcid.org/0000-0002-2362-5308.

Artículo recibido el 22 de mayo de 2020 y aceptado para su publicación el 12 de marzo de 2021. 


\section{INTRODUCCIÓN}

Zn los últimos años en Chile ocurrieron importantes escándalos de corrupción Cque provocaron un fuerte impacto en la opinión pública. Entre ellos, los casos Penta (2014) y Soquimich (2015), relacionados con el financiamiento ilegal de la política; Caval (2015) que involucró al hijo de la presidenta Bachelet en tráfico de influencias; Milicogate (2015) que implicó a funcionarios del Ejército en la desviación de recursos fiscales, y los de colusión de grandes empresas en áreas como la del papel (2016) y de los supermercados (2017). De acuerdo con Latinobarómetro (2016), por primera vez desde 2003, año en que se comenzó a medir este ítem, la corrupción está entre los problemas que más preocupan a los chilenos, siendo superado solo por la delincuencia. Según Transparency International (2017), el 80 por ciento de los chilenos cree que la corrupción ha aumentado mucho y que las autoridades no han hecho un buen trabajo para combatirla.

Dentro de estos escándalos, uno muy significativo fue el ocurrido en Carabineros de Chile, una de las instituciones con mayor confianza ciudadana desde el retorno de la democracia (Latinobarómetro, 2017). El denominado caso Pacogate (2017), como lo llama la prensa en atribución a que a la policía de este país se le apoda de manera coloquial como "pacos", no sólo sumó más de cuatro millones de dólares de desfalco al fisco, sino que involucró a altos mandos de la institución. De acuerdo con Latinobarómetro (2017), si se contabilizan los niveles de confianza de los chilenos en Carabineros de Chile desde 1995 hasta 2017, es este último año el de menor confianza y mayor desconfianza de toda la serie.

Esta investigación aborda el caso Pacogate desde la perspectiva del éscandalo mediático. Así, si el escándalo es la puesta social del acto corrupto, los medios de comunicación con sus determidadas lógicas mediales desempeñan un papel fundamental al exponerlo a la sociedad (Rose-Ackerman y Palifka, 2016; Thompson, 2001).

Ante este panorama, el estudio de los encuadres o frames mediáticos de la corrupción cobra suma relevancia, considerando tanto sus implicaciones en la opinión pública (Entman, 2012; Zamora y Marín Albaladejo, 2010; Park, 2012) como en la rendición de cuentas del poder en los sistemas democráticos (Christians et al., 2009; Coronel, 2010; McQuail, 2013; Waisbord, 2000, 2012; Tagle, 2017a).

Sin embargo, en la actualidad no se puede desconocer la cada vez más relevante importancia e influencia de las redes sociales en el debate público (Keane, 2011). Respecto a ellas, la literatura señala que aportan a la discusión una dimensión polarizante (Cacciatore et al., 2016) y crítica (Ceron, 2015).

En la actualidad, al estudiar los escándalos mediáticos, parece resultar propicio no sólo abocarse en los medios de comunicación tradicionales, como los 
noticieros de televisión, sino que para una mayor comprensión del fenómeno, sumar a las redes sociales, y en este caso, a través de la segunda pantalla que abre nuevas y novedosas formas de participación política (Courtois y D’heer, 2012). Las redes sociales no necesariamente se sobreponen a los medios tradicionales, sino que tienden a complementarse con ellos, especialmente con la televisión y sus noticieros (Weeks et al., 2017; Youyou et al., 2015; Nölleke et al., 2017; Trilling, 2015; Aguilar et al., 2015; Vargo et al., 2015). Se debe destacar que el uso de las plataformas sociales en Chile es elevado, contando Facebook con más de diez millones de usuarios y Twitter sobrepasando los dos millones y medio (Hootsuite, 2020).

Desde la politología, esta investigación se inserta en la rendición de cuentas que puede exigir la sociedad a través de actores sociales como los medios de comunicación a las instituciones de poder en las democracias (Peruzzotti, 2019; Przeworski, 2006; Peruzzotti y Smulovitz, 2002, 2006; O’Donnel, 2004).

Entre los hallazgos de esta investigación se postula como parte del mismo fenómeno mediático que mientras los noticiarios promovieron con sus marcos una definición y explicación del caso, las opiniones de las redes sociales no sólo fueron polarizadas, sino que también dieron una dimensión moralizante y crítica de lo ocurrido. Así, este estudio es una contribución a la comprensión de cómo los medios de comunicación tradicionales y las redes sociales aportan al debate de la opinión pública y a la rendición de cuentas que les exige la ciudadanía a las instituciones en las democracias.

\section{LA RENDICIÓN DE CUENTAS MEDIÁTICAS Y SUS ENCUADRES A LA CORRUPCIÓN}

Si bien ha recibido nuevas formulaciones, la función normativa de los medios como vigilantes del poder es propuesta en la actualidad como una de las principales herramientas de las democracias para que los ciudadanos participen de manera informada en ellas (Christians et al., 2009; Hampton, 2010). Así, McQuail (2013: 98) ha definido como un "nido de cuervo" en el mástil de un barco esta función mediática, haciendo analogía de que los medios son un puesto de observación que alerta de los peligros y riesgos que existen en el horizonte para los ciudadanos.

Desde enfoques politológicos, esta función se ha teorizado como rendición de cuentas social o accountability social, haciendo referencia a que la rendición de cuentas en una democracia puede ser llevada más allá de las instituciones del Estado, estableciéndola también como una relación horizontal con el poder, que proviene desde actores sociales y, en este caso, respecto al actuar vigilante de los 
medios (Fox, 2015; O’Donnell, 1996, 2004). Así, los medios de comunicación con sus denuncias y coberturas de los casos de corrupción ponen estas problemáticas en la agenda de la opinión pública, la que ahora informada puede castigar con su voto a las autoridades que no han realizado su labor de manera adecuada (rendición de cuentas vertical) y presionar a otras instituciones del Estado a que fiscalicen a los actores u organismos corruptos (rendición de cuentas horizontal) (Peruzzotti y Smulovitz, 2002, 2006; Peruzzotti, 2019).

En definitiva, si la racionalidad y la acción de la política llevan a la construcción de instituciones funcionales a las ideas, los valores y las aspiraciones de la sociedad (Auat, 2003), desde la función normativa de la vigilancia mediática se podría argumentar que los medios se preocuparían de asegurar que esas instituciones cumplan la misión para la que fueron creadas. Como explica Keane (2011: 5-6), en las actuales democracias, el afán de monitoreo del poder ha aumentado dado que los ciudadanos "aprovechan dispositivos de control como los medios de comunicación, haciendo que los representados vigilen a sus representantes, mediante críticas y demandas". Así, una de las más clásicas y esperables funciones de los medios tradicionales en la sociedad, que es la vigilancia del poder (Curran, 2002; McQuail, 2013; Waisbord, 2000, 2012; Tagle, 2017a), se ha complementado con el uso de los ciudadanos de Internet, en especial de las redes sociales, lo que les permite ser a ellos protagonistas del escrutinio público al poder (Feenstra y Casero-Ripollés, 2014).

Sin embargo, se debe considerar que las democracias y el buen funcionamiento de su gobernabilidad no son ajenos a las percepciones de la corrupción que tiene la ciudadanía de sus autoridades e instituciones. De acuerdo con Rose-Ackerman y Palifka (2016), el acto corrupto en sí mismo y la justicia van por una vía y la percepción ciudadana de la corrupción por otra, y no necesariamente coinciden. Esta diferencia se explica debido a que, generalmente, la opinión pública accede a una información mediatizada de la corrupción (Thompson, 2001).

De esta manera, cobra relevancia la función de encuadrado de los medios de comunicación (McCombs, 2006; McCombs y Shaw, 1972; McCombs et al., 2014; Cacciatore et al., 2016; Iyengar y Simon, 1993; Scheufele y Tewksbury, 2007; Scheufele e Iyengar, 2012). De modo general, y utilizando la clásica definición de Entman (1993), el encuadrado trata acerca de que los medios de comunicación no sólo ponen temas en la agenda de la opinión pública, sino que también brindan un contexto a partir de la selección de algunos aspectos de la realidad, de manera que se promueva tanto una definición del problema determinado como una interpretación. Así, y como explica Castells (2009: 218), el 
enmarcado mediático funciona dejando vacíos en la información que la audiencia rellena con sus esquemas preconcebidos, "estos son procesos interpretativos de la mente humana basados en ideas y sentimientos conectados, almacenados en la memoria".

Esta visión de la realidad de los medios, como explican Sádaba et al. (2012), hace aceptar que la objetivad periodística al momento de informar nunca estará exenta de determinados enfoques. En esta línea, existen marcos temáticos y episódicos, donde los primeros son los que promueven una explicación estructural del problema, mientras que los segundos lo reducen exclusivamente al suceso (Iyengar, 1991). Es preciso señalar que estudios de los últimos años han reforzado esta tesis, estableciendo que especialmente la televisión encuadra la mayor parte de sus noticias de manera episódica, en asuntos tales como la violencia (Easteal et al., 2015; Larsen, 2018; Falkheimer y Olsson, 2015; Holody y Daniel, 2017), la salud (Gearhart y Dinkel, 2016; Myrick et al., 2014), entre otros.

En lo que se refiere a los escándalos de corrupción, la literatura revisada no brinda estudios de casos sobre encuadres temáticos y episódicos; sin embargo, Park (2012) señala que:

La cobertura de los medios tiende a emplear el enfoque episódico, ignorando el contexto más amplio en el que escándalo de corrupción ha ocurrido. Este sesgo lleva a la ilusión de que los problemas de corrupción desaparecerán cuando los autores sean condenados. Y oscureciendo las conexiones entre un escándalo de corrupción individual y su contexto de ocurrencia, las noticias episódicas trivializan el discurso público sobre las razones fundamentales del escándalo (Park, 2012: 20).

Más allá de los marcos temáticos o episódicos, el estudio de los encuadres mediáticos específicos de la corrupción cobra suma relevancia (Entman, 2012; Tagle, 2021; Tagle y Claro, 2018; Echeverría et al., 2021; Zamora y Marín Albaladejo, 2010; Park, 2012), considerando tanto sus implicaciones para el debate en la opinión pública como para la rendición de cuentas del poder en democracia (Christians et al., 2009). Así, y dado que los encuadres tendrían el poder para estructurar el pensamiento de la opinión pública, es que los medios "suelen proporcionar una cierta interpretación de la corrupción que influye en la forma en que se discute y evalúa en el debate público" (Park, 2012: 3).

De esta manera, al estudiar los encuadres mediáticos de casos de corrupción, cobra relevancia la figura del escándalo político, en cuanto a que es la manifestación social del acto de corrupción (Thompson, 2001). Así, cuando los medios prestan atención a algún hecho ilícito, estos aplican unos marcos que presentan la 
corrupción como algo que desafía las normas existentes y necesita una solución (Entman, 2012) o como una suerte de drama en la que se incluyen villanos y se termina con una moraleja (Zamora y Albaladejo, 2010). Como señalan Clemente y Gabbioneta (2017: 287), los medios con sus encuadres de la corrupción "influyen en la percepción de una transgresión, su magnitud y consecuencias".

A partir de los clásicos encuadres genéricos presentes en las noticias propuestos por Semetko y Valkenburg (2000): a) atribución de responsabilidad, b) conflicto, $c$ ) económico, $d$ ) interés humano y e) moralidad, y como instrumento que se aplicará en esta investigación, Entman (2012) establece que existen cuatro tipos de marcos específicos que mayoritariamente los medios utilizan al momento de encuadrar los escándalos:

1. Definición del problema: El acto corrupto es definido como un problema que impide la correcta operacionalidad del gobierno o de la sociedad.

2. Análisis de las causas: Se atribuye este comportamiento corrupto a un individuo o institución en particular.

3. Juicio moral: El corrupto y el acto corrupto reciben una condenatoria moral pública.

4. Aplicación de un remedio: Se busca una solución que involucre un castigo.

Estos encuadres, por lo general, no se utilizan al mismo tiempo en la cobertura mediática, sino de manera paulatina, respondiendo a un proceso temporal, en el que primero los medios definen el escándalo, luego analizan sus causas, después lo enjuician, para dar finalmente soluciones para que no vuelva a ocurrir lo sucedido.

\section{LAS OPINIONES DE LAS REDES SOCIALES SOBRE LA CORRUPCIÓN}

Las redes sociales permiten una nueva forma de participación, en la que los ciudadanos pueden consumir, distribuir y comentar noticias e información política (Gil de Zúñiga et al., 2014), lo que a su vez estimula que surjan nuevos líderes de opinión política vinculados al mundo online (García-Perdomo, 2017; Weeks et al., 2017).

Si bien una minoría de los usuarios de las redes sociales son los que participan emitiendo sus opiniones, ya sea de manera explícita o compartiendo información, se presume que el resto - que es la gran mayoría— solo leen y observan, pero no contribuyen (Weeks et al., 2017). No obstante, es esta minoría opinante la que tiene un rol protagónico en los comportamientos mediáticos en las redes sociales, al generar debates y animar a otros a expresar sus pareceres. 
Sin embargo, la relación entre las redes sociales y la democracia no necesariamente se caracteriza por ser benigna. Al contrario, quienes opinan en las redes sociales crean una imagen que les resulta coherente con la que tienden a polarizar los marcos que reciben desde los medios tradicionales (Cacciatore et al., 2016), y en vez de fomentar el diálogo en muchas ocasiones son espacios para expandir los miedos (Youyou et al., 2015).

Una de las principales caraterísticas de las redes sociales, como Facebook o Twitter, es que permiten la comunicación de manera horizontal y entre pares, lo que ayuda a enriquecer el debate democrático de las sociedades (Keane, 2011; Meraz, 2009; Ceron, 2015). Por ejemplo, como señalan García et al. (2017), el clima de opinión en redes sociales como Twitter contribuye a la creación de una agenda complementaria a la de los medios tradicionales. En este sentido, aunque las redes sociales se nutren de las noticias de la prensa, la radio y la televisión, la rápidez y abundancia de información provoca que se genere su propia lista de asuntos de interés. Esto conlleva a plantear, como explican Grundberg y Lindgren (2015), que las redes sociales influyen con sus propios marcos al debate democrático.

Por otra parte, la natruraleza no mediada de las redes sociales permite a los ciudadanos acceder de manera directa a la información, sin necesariamente el control centralizado de las élites sobre el discurso, dando cabida a posturas críticas y antisistema (Hermida, 2010; Bailard, 2012). No obstante, no actúan en una esfera pública distinta a la de los medios tradicionales, al contrario, existe una lógica híbrida de la comunicación política (Chadwick, 2013), donde las redes sociales se alimentan de las noticias de los medios tradicionales, y estos seleccionan e incorporan algunos mensajes de los ciudadanos en el mundo online para su propia agenda.

Es importante señalar que Facebook y Twitter tienen naturalezas distintas; mientras la primera está orientada a las amistades, la segunda tiene una carácter de discusión política (García et al., 2017). Sin embargo, las características de Facebook no lo hacen ajeno al debate político, sino que se produce de manera distinta. Por una parte, esta red social promueve una mayor confianza al momento de opinar, dado que se trata de un intercambio entre conocidos (Jacobson, 2013), y por otra, los estudios indican que los medios la utilizan para difundir sus noticias y redirigir a los usuarios al sitio nativo (Jung y Villi, 2018).

En lo que se refiere a los escándalos de corrupción, Jha y Sarangi (2017) señalan que las redes sociales no sólo ayudan a exponer los ilícitos a una mayor audiencia, sino que dan cercanía al problema, transformándolo en una realidad que puede afectar al ciudadano común. 
De acuerdo con Ceron (2015), en el tratamiento de los escándalos de corrupción, los medios de comunicación tradicionales no sólo informan de manera crítica, sino que dan cobertura a cómo el sistema democrático afronta la crisis y sale de ella (actuar de la justicia, nueva legislación, etc.) promoviendo una suerte de "círculo virtuoso". En cambio, en las redes sociales el resarcimiento de las instituciones o de los culpables parece más oculto, y priman la desconfianza y los altos estándares de exigencia. La contracara de este fenómeno, de acuerdo con McHale (2017), es que las redes sociales pueden promover un ambiente pernicioso para el sistema político, al caer en generalidades e incluso fantasías - fake news - sobre las reales dimensiones de la corrupción.

Por otra parte, las redes sociales también pueden cumplir la función de hacer rendir cuentas al poder, al promover el descontento y hacer que las instituciones encargadas de impartir justicia actúen (Perruzotti y Smulovitz, 2006). En este sentido, como señalan Prabowo et al. (2018), las redes sociales pueden crear un entorno social hostil para los corruptos, haciendo de la corrupción una elección más irracional. En esta línea, como señala un estudio de Elbahnasawy (2014), los países con un mayor uso de las redes sociales tienden a tener una menor percepción de la corrupción.

\section{COMPLEMENTARIEDAD DE LOS NOTICIEROS Y LAS REDES SOCIALES: SEGUNDA PANTALLA}

Los usuarios de las redes sociales utilizan los contextos y sugerencias de la cobertura de los medios tradicionales como una pauta para procesar la información (Kepplinger et al., 2012; Gibs et al., 2009). Esto ha abierto nuevas formas de consumo de los medios tradicionales. Como señalan Courtois y D’heer (2012), en los últimos años hay una tendencia de los usuarios de Facebook o Twitter a mirar televisión con un teléfono inteligente o tablet desde donde simultáneamente opinan del contenido televisivo, lo que ha sido denominado como segunda pantalla o second screen.

Es este sentido, no se trata de un fenómeno menor o aislado, al contrario, de acuerdo con un estudio de Rosenstiel et al. (2015), de 4700 usuarios de Twitter en Estados Unidos, más de 50 por ciento comentaba el contenido ofrecido por las noticias de la televisión. La razón de este fenómeno, según Neate et al. (2017), es que la televisión ofrece una gran interacción social, da un punto de conversación común y reúne a las familias en torno a ella, lo que ha sido aprovechado por las redes sociales para integrarse a ella comentando sus contenidos.

No obstante, esta interacción no se trata de una relación exclusivamente unidireccional, donde las redes comentan el contenido televisivo, sino también los 
mismos comentarios son recogidos por los espacios televisivos y los suman algunas veces a su contenido (Trilling, 2015).

Respecto a los noticieros, los usuarios de una segunda pantalla a partir del contenido televisivo que se está transmitiendo buscan de manera simultánea reflexionar, discutir y confirmar opiniones con otros. De acuerdo con Chadwick et al. (2017), se pueden dividir en dos tipos a quienes utilizan la segunda pantalla para la participación política. Por una parte, están los que intentan influir en los propios periodistas que producen la noticia y, por otro, los que desean compartir información y opinión para influir en sus propios seguidores y usuarios en general.

Es así como el uso de la segunda pantalla tendría un aspecto positivo para el debate en democracia, dado que serviría como una suerte de mediador crítico y simultáneo entre las noticias de los medios tradicionales y los ciudadanos, lo que ofrece una nueva forma de interacción en la opinión pública (Gil de Zúñiga et al., 2015). Cabe destacar que las principales investigaciones en el ámbito político del uso de la segunda pantalla han sido sobre su utilización en los debates políticos televisados (Gorkovenko y Taylor, 2016; Jensen, 2016).

Existe una tendencia en los países con una menor confianza en las instituciones (Transparency International, 2019) a un mayor uso de la segunda pantalla. Un estudio de Gil de Zúniga y Liu (2017) concluye que en las naciones con un periodismo más precario o con un mayor nivel de corrupción tiende a existir una mayor utilización de la second screen, lo que promueve una suerte de periodismo ciudadano instantáneo, en respuesta a las instituciones que no generan confianza. Por ejemplo, en países como Turquía, China, Filipinas y Brasil, el uso de la segunda pantalla es mayor que en Estados Unidos o Alemania.

\section{METODOLOGÍA}

El escándalo Pacogate estalló el 6 de marzo de 2017 cuando los medios de comunicación informaron sobre un millonario fraude al interior de Carabineros de Chile. En una serie de operaciones ilícitas que comenzaron en 2010, y se extendieron hasta finales de 2015, funcionarios de la institución acumularon un patrimonio de más de 15 millones de dólares. Por este ilícito se han imputado 115 carabineros, de los que 33 pertenecen al alto mando y uno de ellos es un general.

A partir del ciclo de encuadres mediáticos propuestos por Entman (2012) respecto a los escándalos de corrupción, y teniendo como caso de estudio los noticiarios de televisión y las opiniones vertidas en Facebook y Twitter respecto al caso Pacogate en Chile, esta investigación se fundamenta en las siguientes hipótesis: 
Hipótesis 1: Los noticiarios de televisión y las redes sociales Facebook y Twitter promovieron diferentes marcos de corrupción, permitiendo una complementariedad entre la cobertura de los noticiarios y las opiniones de las plataformas digitales.

Hipótesis 2: Existen diferencias entre las redes sociales Facebook y Twitter, en uso de la segunda pantalla, respecto a las opiniones del escándalo de corrupción.

El periodo seleccionado es desde el 6 de marzo de 2017, fecha en la que estalló el escándalo, hasta los siguientes 14 días consecutivos. De acuerdo con Entman (2012), al menos una semana es un periodo oportuno para analizar las implicaciones de un escándalo mediático.

Para el análisis de la televisión, se seleccionaron los noticiarios centrales de las 21:00 horas, con mayor sintonía, los que para el periodo de estudio fueron Ahora Noticias y Teletrece de las estaciones privadas Mega y Canal 13 respectivamente (Kantar, 2017).

A partir de los encuadres descritos por Entman (2012), sobre cómo encuadran los medios de comunicación los escándalos de corrupción, se enmarcó y midió en minutos cada noticia. Se prefirió utilizar los cuatro encuadres de Entman (2012) por sobre otras propuestas teóricas de marcos específicos para la corrupción debido a que son solo cuatro marcos de definición amplia — por ejemplo, $\mathrm{Za}$ mora y Albaladejo (2010) proponen diez encuadres-, lo que permite una fácil sistematización de aplicabilidad a un caso de estudio.

Dado que la unidad de análisis de esta investigación es la noticia, se contabilizaron todas las notas aparecidas en ambos noticieros que hubieran hecho mención al caso Pacogate. La muestra fue enmarcada por dos codificadores, a quienes previamente se les dieron a conocer los marcos de Entman (2012) y los alcances del encuadrado, poniendo también en común, de acuerdo con Tankard (2001), que tras el análisis de todos los elementos que constituían las noticias, desde los titulares hasta sus imágenes, la idea central que se promovía debía ser considerada como el marco predominante.

Por otra parte, se realizaron sumatorias de los espacios dedicados a cada marco en ambos noticiarios, con el propósito de establecer comparaciones respecto a los cuadros que primaron en cada medio.

En lo que se refiere a las opiniones vertidas en Facebook y Twitter, se utilizó una plataforma comprehensiva de captura en tiempo real de la información en correlación aproximada al horario de los noticiarios centrales (21:00 horas), la cual fue brindada por la empresa chilena de análisis online Brandemetric. De esta 
manera, se recogieron todos los comentarios de ambas redes sociales que tuvieran la palabra clave "Pacogate". Una vez recogidas todas las opiniones, estas se clasificaron según los encuadres de la corrupción utilizados en los medios.

Dado que esta investigación se enfocó en cómo encuadran las redes sociales de manera general en cuanto al fenómeno de su complementariedad con los noticiarios de televisión, no se hace distinción entre los diferentes usuarios que las utilizan, ya sea por edad o por género, o si se trata de políticos, periodistas o ciudadanos comunes.

De los 14 días analizados, el total de la muestra televisiva correspondió a 24 unidades informativas entre los dos medios televisivos. Así, se identificaron 11 noticias en Ahora Noticias, correspondientes a 34 minutos en total, y 13 en Teletrece, las cuales equivalieron a 59 minutos.

Por su parte, en las redes sociales se recogieron 5882 menciones referidas al caso. De estas menciones, 56 por ciento se realizó en Twitter y 44 por ciento en Facebook, todas geolocalizadas en territorio chileno.

Respecto a la fiabilidad de los resultados, las noticias y los comentarios de las redes sociales fueron codificados por dos jueces independientes, bajo el diseño de reproducibilidad de los datos, que incluyó un retest de la muestra de 10 por ciento (Krippendorff, 1990). De esta manera, el coeficiente de confiabilidad fue de 0.9.

\section{RESULTADOS}

Mientras en los noticieros los marcos predominantes fueron definición del problema y análisis de las causas, en las redes sociales la clasificación principal de los comentarios correspondió al encuadre moralidad.

CUADRO 1. Porcentajes de la cantidad total de minutos dedicados al caso Pacogate en los noticiarios Ahora Noticias y Teletrece del 6 al 19 de marzo de 2017 según los encuadres de corrupción de Entman (2012)

\begin{tabular}{lcc}
\hline & Teletrece (\%) & Ahora Noticias (\%) \\
\hline Definición del problema & 23 & 52 \\
Análisis de las causas & 77 & 48 \\
Juicio moral & 0 & 0 \\
Aplicación de un remedio & 0 & 0 \\
Total & 100 & 100 \\
\hline
\end{tabular}

Fuente: Elaboración propia. 
CUADRO 2. Porcentajes de la cantidad total de comentarios dedicados al caso Pacogate en las redes sociales Facebook y Twitter del 6 al 19 de marzo de 2017 clasificadas temáticamente, según los encuadres de corrupción de Entman (2012)

\begin{tabular}{lcc}
\hline & Facebook (\%) & Twitter (\%) \\
\hline Definición del problema & 0 & 0 \\
Análisis de las causas & 0 & 0 \\
Juicio moral & 91 & 89 \\
Aplicación de un remedio & 9 & 11 \\
Total & 100 & 100 \\
\hline
\end{tabular}

Fuente: Elaboración propia.

GRÁFICA 1. Porcentajes de los marcos de los noticiarios y la clasificación de los comentarios de las redes sociales respecto al caso Pacogate

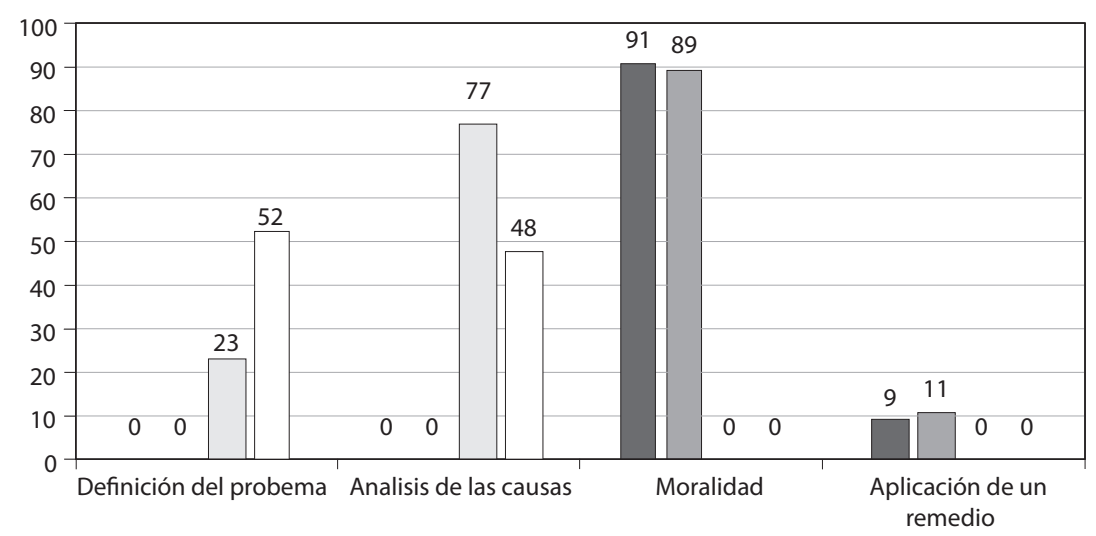

$\square$ Facebook $\square$ Twitter $\square$ Teletrece $\quad \square$ Ahora Noticias

Fuente: Elaboración propia.

Esto lleva a plantear que no existe una superposición de los encuadres de Entman (2012) entre los dos noticiarios de televisión y Facebook y Twitter. Al contrario, se muestra, usando un mismo criterio de análisis, que los programas televisivos promueven determinados marcos, mientras que las redes otros.

En la gráfica 1 se presenta una combinación de todos los resultados, en donde se aprecia una predominante tendencia en los dos noticiarios a encuadrar en 
los mismos marcos. Una situación similar ocurre con las dos redes sociales y las clasificaciones preponderantes de los comentarios.

\section{LOS MARCOS DE LOS NOTICIEROS: DEFINICIÓN DEL PROBLEMA Y ANÁLISIS DE LAS CAUSAS}

Si bien ambos noticiarios encuadraron en los mismos marcos, existe diferencia respecto a que las noticias de Teletrece utilizaron mayormente el marco de análisis de las causas, mientras que Ahora Noticias enmarcó principalmente en definición del problema. Investigaciones recientes han establecido que los noticiarios chilenos estereotipan la pobreza (Etchegaray y Matus, 2015) y los rasgos étnicos (Amigo et al., 2016), siendo Teletrece uno de los noticiarios menos sensacionalistas, mientras que Ahora Noticias, uno de los más (Celis Fernández, 2017). Esto último podría explicar por qué Ahora Noticias tuvo un mayor afán en centrarse en el escándalo en cuanto tal, mientras que Teletrece intentó explicar y analizar el poqué se produjo.

Como señala Entman (2012), los medios de comunicación encuadran primeramente definiendo el acto de corrupción como un problema que impide el buen funcionamiento de la sociedad. En el caso Pacogate, los dos noticieros promovieron la anormalidad de lo que estaba sucediendo con la policía (Ahora Noticias, 2017a, 2017b, 2017c; Teletrece, 2017a, 2017b, 2017c).

Si bien como se ha mencionado, el escándalo de corrupción es la sociabilización del acto corrupto (Thompson, 2001), no se debe dejar de considerar que este también permite el cumplimiento de una de las funciones más esperables de los medios de comunicación en democracia: la función de vigilancia, informando a la ciudadanía sobre el actuar de quienes ostentan el poder, como señalan autores como Waisbord (2000; 2012), Peruzzotti y Smulovitz (2006) y Tagle (2017a). Si bien Pacogate, a diferencia de otros casos de corrupción, no nació a partir de una investigación periodística de los medios analizados, la propia cobertura de lo ocurrido y el afán de denuncia se pueden considerar como parte del papel de la vigilancia mediática (Tagle, 2017a).

Los noticiarios no sólo informaron de lo que estaba ocurriendo, sino que intentaron analizar las causas de lo sucedido al interior de Carabineros, pero no de manera estructural ni moral, sino en cuanto a las falencias del sistema y la astucia de los corruptos.

Es que se siguen conociendo detalles lo que ya sería una investigación sumaria. Según el abogado de uno de los oficiales desvinculados, la operación de estos funcionarios era la siguiente: uno de ellos prestaba su cuenta corriente para recibir depósitos que tendrían que ver con glosas asociadas a bonos y viáticos, de ese dinero, el titular de la cuenta se quedaría 
con 10 por ciento de lo defraudado, el resto era repartido en efectivo entre los oficiales desvinculados de la institución.

Una de las falencias aprovechadas es que nadie fiscalizaba el reintegro de esos dineros. Según el teniente coronel Mauricio Saldaña Vidal, uno de los oficiales expulsados, él habría recibido, desde 2013, la suma de $\$ 300$ millones, en depósitos fragmentados que podían alcanzar fácilmente hasta los $\$ 50$ millones. Es así como la malversación de fondos fiscales llegaría hasta ahora a los $\$ 8$ mil millones, según el mismo general director de Carabineros. (Teletrece, 9 de marzo de 2017).

Sin embargo, este análisis de las causas no se redujo al caso en sí mismo, sino que se amplió al actuar del Poder Judicial, como una institución ineficiente en su labor de impartir justicia. Por ejemplo, Ahora Noticias dio cobertura al control de detención de los sospechosos y cómo los tribunales los dejaron en libertad sin medidas cautelares:

Cuatro de la mañana y los 17 carabineros investigados por el millonario fraude al interior de la institución salieron del Centro de Justicia sin ninguna medida cautelar en su contra. No tienen arresto domiciliario, ni siquiera arraigo nacional (Ahora Noticias, 13 de marzo de 2017).

Resulta interesante esta segunda dimensión del encuadramiento de análisis de las causas, esto teniendo presente que el Poder Judicial es una de las instituciones del Estado chileno con menor confianza ciudadana (Latinobarómetro, 2017). Como señalan autores como O'Donnell (1996; 2004) y Peruzzotti y Smulovitz (2006), en América Latina actores sociales, como los medios de comunicación, han tenido un papel importante para asegurar que las instituciones del Estado cumplan de manera más eficiente su función.

Sin embargo, y como señalan Zamora y Albaladejo (2010), la mediatización del acto corrupto lleva generalmente consigo un drama, donde existen inescrupulosos villanos. Los noticiarios también promovieron una definición del acto corrupto a partir de la personificación y de la vida de lujo que estaban llevando los culpables antes de ser sorprendidos. Por ejemplo, Teletrece a partir de una investigación propia, denunció el estilo de vida que llevaban algunos de los carabineros involucrados en el millonario desfalco.

Septiembre de 2013 y la pareja del teniente coronel, Héctor Nail Bravo, agradece a través de Facebook al "salvador" Robinson Carvajal Leiva por la gestión que hizo para concretar un paseo en moto al Embalse el Yeso. Viaje que no era más que una reunión de amigos que hoy cobra relevancia, porque ahí están al menos tres de los supuestos implicados en esta red [...] 
En las fotos no aparecen sus bienes, pero Teletrece indagó en las propiedades que posee y se pudo consignar que tiene dos viviendas que suman cerca de $\$ 300$ millones como avalúo, además de dos vehículos (Teletrece, 12 de marzo de 2017).

Un aspecto que se debe destacar dentro del análisis, y siguiendo las clasificaciones de encuadre de Iyengar (1991) sobre marcos temáticos y episódicos, es que ninguna de las noticias analizadas en ambos noticiarios encuadró de manera temática el escándalo. No se encontraron conexiones con otros casos de corrupción o que se promoviera una problematización de lo ocurrido como un fenómeno estructural de la sociedad chilena.

\section{LOS COMENTARIOS EN LAS REDES SOCIALES: MORALIDAD Y APLICACIÓN DE UN REMEDIO}

Resulta interesante comprobar que mientras en los noticiarios el escándalo Pacogate se redujo casi exclusivamente al hecho en sí mismo y a sus causas, fueron las opiniones en las redes sociales las que conectaron el caso con dimensiones que iban más allá de lo propiamente sucedido. Mientras que en los noticiarios el encuadre de juicio moral no se presentó, en las redes sociales fue el marco preponderante.

De acuerdo con un estudio de Valenzuela et al. (2018) sobre la utilización de redes sociales en Chile, tanto Twitter como Facebbok tienen efectos positivos para la participación política. Sin embargo, las opiniones vertidas en Facebook tienen mayores vínculos emocionales, lo que puede promover una mayor reflexión por parte del receptor. Esto podría explicar en esta investigación que, si bien los resultados de los encuadres en ambas redes sociales son bastante similares, hay una tendencia en Facebook a categorizar más en el marco de juicio moral, dado que apela mayormente a las emociones.

Por otra parte, Twitter ha demostrado ser un importante medio de comunicación ciudadano en los desastres naturales que frecuentemente ocurren en Chile (Morales et al., 2018; Tagle, 2017b) y de protesta política (Valenzuela et al., 2018). Lo anterior lleva a plantear, para esta investigación, que la mayor tendencia de Twitter a enmarcar en aplicación de un remedio se debe a su utilización como un medio de información y de debate político.

De manera general, se pudo establecer que el juicio que hacen las redes sociales abarca distintos ámbitos, que van desde opiniones con miedo y rabia, pero no por ello, menos moralizantes, hasta propósitos de no generalizar, comparaciones con otros escándalos y establecer a la corrupción como un problema sistémico de la sociedad. 
Como señalan Youyou et al. (2015), Facebook y Twitter se han convertido en ventanas a través de las cuales las personas expresan y exponen no solo sus opiniones, sino también sus temores. Las opiniones en las redes sociales fueron en alusión a exagerar la situación de crisis y a dramatizar lo que estaba ocurriendo como un problema que abarca a todo el país: "Ahora sí que nos quedamos con cero institución confiable" (Twitter, 07 de marzo de 2017), "Ya no existen instituciones libres de corrupción" (Twitter, 08 de marzo de 2017), "Chile no es un país corrupto, decían" (Twitter, 08 de marzo de 2017), "Ya no se puede confiar absolutamente en nada" (Twitter, 07 de marzo de 2017), "Y yo que orgulloso decía a los extranjeros que los carabineros de Chile eran una institución admirable, sin corrupción y honorable... al final son todas iguales, no se puede confiar en nadie" (Facebook, 10 de marzo de 2017).

Kepplinger et al. (2012) señalan que hay una tendencia a la radicalización en los comentarios de las redes sociales, por lo que un escándalo de corrupción del poder político sería un buen motivo para fomentarla. Así, el juicio moral de las opiniones tanto en Facebook como en Twitter vino acompañado de expresiones de enojo y rabia ante el escándalo, las cuales incluyeron también garabatos y términos vulgares: "CaraDineros de Chile" (Twitter, 11 de marzo de 2017), "Me va a dar rabia cuando vea a un carabinero subiendo sin pagar a la micro" (Facebook, 09 de marzo de 2017), "Pacos culiaos le roban al Estado!!!, milicos culiaos parásitos del Estado!!!, Chupasangres!!!” (Twitter, 11 de marzo de 2017), "Pacos punga, políticos corruptos, autoridades incompetentes, justicia no hace su pega" (Twitter, 12 de marzo de 2017), iii “Pacos corruptos de la стм!!!” (Twitter, 10 de marzo de 2017), "Estos hijos de puta hacen lo que quieren" (Facebook, 12 de marzo de 2017).

Como se señaló en los apartados anteriores, Carabineros de Chile es una de las instituciones mejor valoradas por los chilenos desde el retorno de la democracia (Latinobárometro, 2019), lo que se demostró en que hubo opiniones en las redes sociales que intentaron personificar el caso solamente en los funcionarios corruptos diferenciándolos del resto de la institución: "Es una institución tan grande y bien ponderada por la ciudadanía, no debe culparse por unos pocos malos elementos" (Twitter, 11 de marzo de 2017), "Lamentable que unos pocos enloden a toda la Institución” (Twitter, 10 de marzo de 2017). Al contrario, se intentó personificar la corrupción sólo en la oficialidad y en los altos mandos: "Los verdaderos \#CarabinerosdeChile son los que están en la calle día a día cumpliendo el juramento que hicieron" (Twitter, 12 de marzo de 2017). "Los errores los comenten personas...no instituciones y los oficiales que la cagaron se tienen que ir pero no son todos los carabineros" (Facebook, 12 de marzo de 2017). "En una institución 
tan grande y bien ponderada por la ciudadanía que no debe culparse por unos pocos malos elementos en los altos mandos" (Facebook, 11 de marzo de 2017).

Otro ámbito del marco moralidad fue el de conectarlo con otras problemáticas de la sociedad chilena que carecen de recursos: "Una pena que se gasten la plata de los chilenos en darse gustos mientras nuestros compatriotas luchan por ganarse el pan" (Twitter, 07 de marzo de 2017), "Y después salen diciendo por la prensa que no hay plata para educación, vivienda y salud..." (Twitter, 07 de marzo de 2017), "Pensaba que con 8 mil millones cuántos estudiantes podrían estudiar gratis..." (Twitter, 11 de marzo de 2017), "Cuántos aviones para combatir incendios se podrían comprar con 15 mil millones?" (Facebook, 12 de marzo de 2017), "No hay plata para salud, vivienda, educación, claro ya sabemos quiénes se la llevan para su casa" (Twitter, 07 de marzo de 2017).

Por otra parte, al utilizar la definición del encuadre de juicio moral como una categoría de clasificación en las redes sociales, este estuvo presente en cuanto a desconfiar de la justicia, haciendo alusión a que los castigos a los carabineros implicados, por tratarse de una institución de poder, fueron menos severos: "Que el castigo no sea dar de baja...Se nota que hay chilenos que están por encima de la ley..." (Twitter, 08 de marzo de 2017), "Los 9 oficiales atentaron contra la institución... no debieran recibir penas más endurecidas de acuerdo con su propia ley...?" (Twitter, 11 de marzo de 2017)", "El \#pacogate tiene que investigarse y los responsables ir a la cárcel" (Twitter, 07 de marzo de 2017), "Claro, como son oficiales de carabineros no reciben el castigo que merecen, sino que a retiro..." (Facebook, 10 de marzo de 2017), "Queda claro que en Chile los castigos no son iguales para todos" (Twitter, 12 de marzo de 2017).

Sin embargo, y a diferencia de los noticiarios y tomando la clasificación de Iyengar (1991), este juicio moral de las redes sociales fue más allá de un enojo o una lamentación, sino que también fue una comparación con los otros casos de corrupción ocurridos en el último tiempo, dándole a la corrupción una connotación sistemática de la sociedad chilena: "Estamos llenos de \#Gate: \#Pacogate, \#MilicoGate, \#EmpresarioGate, \#PolíticoGate, \#Pescagate”, etc.” (Twitter, 09 de marzo de 2017), "La institucionalidad neoliberal funciona a través de la corrupción, empresarios, políticos, curas, militares y ahora carabineros" (Twitter, 11 de marzo de 2017), "\#Pacogate, \#Milicogate, \#Leyreservadacobre, \#Leydepesca, \#AFP, \#Isapres, son todos narcoooooos!!!!” (Twitter, 12 de marzo de 2017).

De manera bastante menos considerable que juicio moral, en las redes sociales también estuvo presente la clasificación aplicación de un remedio, aunque no fue en cuanto a una solución concreta para que no hubiera nuevos casos, sino una 
crítica a la severidad del castigo: "Llamado a retiro, después seguramente serán culpables, condenados a 60 años de libertad vigilada y gozarán de su jubilación" (Facebook, 10 de marzo de 2017), "Media cosa que los llamen a retiro se van con pensión y con la plata que se robaron" (Twitter, 11 de marzo de 2017), "Cada uno se irá con pensión vitalicia de 3 palitos. Orden y patria es su lema” (Twitter, 12 de marzo de 2017).

\section{CONCLUSIONES}

En el caso Pacogate, que remeció a la opinión pública chilena, por tratarse de la policía, una de las instituciones de mayor credibilidad de ese país, fue posible establecer una suerte de complementariedad entre los marcos que promovieron los noticiarios de televisión y los comentarios de las redes sociales Facebook y Twitter bajo uso de la segunda pantalla.

Los marcos de la corrupción de los medios de comunicación no son inocuos, sino que comunican el acto corrupto a partir de determinados enfoques e interpretaciones (Zamora y Albaladejo, 2010). En esta investigación no sólo se comprobaron los marcos propuestos por Entman (2012) en los noticiarios de televisión chilenos, sino que al llevar estos encuadres al ámbito de las redes sociales fue posible advertir una nueva dimensión de discusión. Así se pudo establecer que las redes sociales, a la vez de polarizar el discurso que reciben de los medios tradicionales, no promoviendo necesariamente el diálogo (McHale, 2017), y ser un lugar para expresar miedos (Youyou et al., 2015), lo que implica los cuestionamientos a su relación con la democracia, también forman parte del propio proceso de mediatización del escándalo de corrupción (Jha y Sarangi, 2017; Ceron, 2015), tendiendo a su complementariedad (Chadwick et al., 2017).

Mientras los noticiarios de televisión promovieron una definición del escándalo Pacogate tanto identificando a los culpables como vigilando el actuar de la Justicia (Curran, 2002; McQuail, 2013; Waisbord, 2000, 2012; Tagle, 2017a), las opiniones de las redes sociales fueron un lugar para brindar un juicio moral y ofrecer una perspectiva más amplia de la corrupción, conectándola con otros casos y estableciéndola como una problemática estructural de la sociedad chilena. Así fue como se pudo establecer, siguiendo la clasificación de Iyengar (1991), que mientras los noticiarios de televisión emarcaron de manera más episódica la corrupción, las redes sociales tendieron a su tematización, abriendo nuevas formas de debate.

En términos de Castells (2009), y en el caso de la corrupción, podemos comprobar que las redes sociales rellenaron de manera crítica el espacio dejado por 
los marcos que promueven los noticieros de televisión. Así, no sólo se demostró que las redes sociales utilizan los medios de comunicación tradicionales como una pauta para procesar información noticiosa (Kepplinger et al., 2012; Gibs et al., 2009), sino que desde la simultaneidad que ofrece la segunda pantalla aportaron nuevas dimensiones para la comprensión del escándalo de corrupción (Jha y Sarangi, 2017). A diferencia de los medios tradicionales, donde el mensaje está subordinado a los límites que le da su propio entorno, en las redes sociales su falta de control centralizado y su propia naturaleza no mediada (Hermida, 2010; Bailard, 2012) pueden permitir un mayor cuestionamiento del escándalo de corrupción y hacer conexiones con otros actos ílicitos que en los medios tradicionales no siempre se pueden presentar.

A pesar de que el estudio esperaba encontrar diferencias entre Facebook y Twitter y su forma de mediar la corrupción (García et al., 2017), solo fue posible vislumbrar una leve tendencia en Facebook al juicio moral, tal vez por su carácter de mayor confianza y emocionalidad, dado que se trata de un lugar de "conocidos" (Valenzuela et al., 2018; Jacobson, 2013). Asimismo, al adentrarnos en las diferencias entre los noticieros se pudo advertir que el considerado más sensacionalista (Celis Fernández, 2017) tuvo una prefencia por reducir el escándalo a los hechos, mientras que el menos sensacionalista promovió una mayor explicación de lo ocurrido.

Desde una perspectiva de la politología, el aporte de esta investigación radica en la mejor comprensión del papel de las redes sociales como posibles actores para la rendición de cuentas social en las democracias. Por otra parte, desde la comunicación, este estudio da pistas de cómo se comportan los usuarios de las redes sociales y cómo interatuán con medios "tradicionales" como los noticiarios de televisión.

De esta manera, y desde el ámbito de la segunda pantalla, se propone para investigaciones futuras abordar nuevos enfoques que se adentren en las diferencias entre Facebook y Twitter al mediar la corrupción y la correspondencia entre noticiarios y las distintas redes sociales respecto al sensacionalismo. También se abre una discusión si el no uso de la segunda pantalla y su simultaneidad permiten de la misma forma o no la complementariedad entre los noticieros y las redes socielas respecto a los escándalos de corrpución.

Entre los límites de esta investigacióm y propuestas para futuras, está la necesidad de nuevos estudios, probablemente de percepciones de la opinión pública, sobre cómo los medios y su cobertura de la corrupción impactan en ella y posteriores acciones de hacer rendir cuentas al poder. 
El estudio de cómo en las sociedades actuales se mediatizan los actos de corrupción política cobra suma relevancia para el funcionamiento de la democracia (Keane, 2011). Así, en la actualidad, para una mejor comprensión del fenómeno del escándalo, parece no bastar con reducir las investigaciones a lo que comunican los medios tradicionales, sino que para tener un panorama completo es indispensable sumar a las redes sociales. G트

\section{REFERENCIAS}

Aguilar, M.G., F.J.P. Rojano y P.F. Batlle (2015), "Comportamiento de la audiencia de televisión en las redes sociales: Una aproximación al perfil y programas más comentados", Revista Latina de Comunicación Social, 70(5), pp. 539-551, Dor: 10.4185/RLCS2015-1058.

Ahora Noticias (2017a), "Carabineros llama a retiro a un general y a ocho oficiales por presunto fraude en el fisco", 10 de marzo, Santiago de Chile, Meganoticias.

Ahora Noticias (2017b), "Urgente: 9 oficiales de Carabineros dados de baja", 6 de marzo, Santiago de Chile, Meganoticias.

Ahora Noticias (2017c), "Ya van 15 funcionarios expulsados", 10 de marzo, Santiago de Chile, Meganoticias.

Amigo, B., M.C. Bravo, C. Sécail, P. Lefébure y A. Borrell (2016), “Televisión, diversidad y hegemonía cultural: Un estudio comparado de los estereotipos étnicos dominantes en los sistemas televisivos de Chile y Francia", Cuadernos.info, 39, pp. 151-164, Dor: http:// dx.doi.org/10.7764/cdi.39.754.

Auat, L.A. (2003), "La racionalidad política: Principios y mediaciones", Tópicos, 11, pp. 45-61. Bailard, C.S. (2012), "Testing the Internet's Effect on Democratic Satisfaction: A Multimethodological, Cross-national Approach", Journal of Information Technology \& Politics, 9(2), pp. 185-204, DoI: https://doi.org/10.1080/19331681.2011.641495.

Cacciatore, M.A., D.A. Scheufele y S. Iyengar (2016), "The End of Framing as We Know It... and the Future of Media Effects", Mass Communication and Society, 19(1), pp. 7-23, DOI: $10.1080 / 15205436.2015 .1068811$.

Casero-Ripollés, A., S. Ortells Badenes y G. Rosique Cedillo (2014), "La espectacularización de la política: Consecuencias democráticas de la disolución de las fronteras entre información, entretenimiento y privacidad en la era digital", Telos: Cuadernos de Comunicación e Innovación, 99, pp. 45-54.

Castells, M. (2009), Comunicación y poder, Madrid, Alianza Editorial.

Celis Fernández, T. (2017), "Detrás del miedo: Análisis de la construcción discursiva del miedo al delito en Chile en los noticiarios centrales de televisión", tesis de maestría, Universidad de Chile. 
Ceron, A. (2015), "Internet, News, and Political Trust: The Difference between Social Media and Online Media Outlets", Journal of Computer-Mediated Communication, 20(5), pp. 487-503, Dor: https://doi.org/10.1111/jcc4.12129.

Chadwick, A. (2013), The Hybrid Media System: Politics and Power, Nueva York, Oxford University Press.

Chadwick, A., B. O'Loughlin y C. Vaccari (2017), "Why People Dual Screen Political Debates and Why It Matters for Democratic Engagement", Journal of Broadcasting \& Electronic Media, 61(2), pp. 220-239, Dor: https://doi.org/10.1080/08838151.2017.1309415.

Christians, C., T. Glasser, D. McQuail, K. Nordenstreng y R. White (2009), Normative Theories of the Media: Journalism in Democratic Societies, Urbana y Champaign, Universidad de Illinois.

Clemente, M. y C. Gabbioneta (2017), "How Does the Media Frame Corporate Scandals? The Case of German Newspapers and the Volkswagen Diesel Scandal", Journal of Management Inquiry, 26(3), pp. 287-302, DOI: https://doi.org/10.1177/1056492616689304. Coronel, S. (2010), "Corruption and the Watchdog Role of the News Media", en P. Norris (ed.), Public Sentinel: News Media and Governance Reform, Washington, D.C., Banco Mundial, pp. 111-136.

Courtois, C. y E. D’heer (2012), "Second Screen Applications and Tablet Users: Constellation, Awareness, Experience, and Interest", Proceedings of the 10th European Conference on Interactive TV and video, ACM, pp. 153-156).

Curran, J. (2002), Media and Power, Nueva York, Routledge.

Easteal, P., K. Holland y K. Judd (2015), "Enduring Themes and Silences in Media Portrayals of Violence Against Women", Women's Studies International Forum, 48, pp. 103-113, DoI: https://doi.org/10.1016/j.wsif.2014.10.015.

Echeverría, M, R. González y F. Tagle (2021), "Corruption Framing in Latin American Media Systems: A Comparison Between Mexico and Chile", The Journal of International Communication, 27(2), pp. 149-171, DoI: 10.1080/13216597.2021.1904268.

Elbahnasawy, N.G. (2014), "E-government, Internet Adoption, and Corruption: An Empirical Investigation”, World Development, 57, pp. 114-126, Dor: https://doi.org/ 10.1016/j. worlddev.2013.12.005.

Entman, R. (1993), "Framing: Toward Clarification of a Fractured Paradigm”, Journal of Communication, 43(4), pp. 51-58.

Entman, R. (2012), Scandal and Silence: Media Responses to Presidential Misconduct, Cambridge, Polity Press.

Etchegaray, N. y A. Matus (2015), "Evolución de la cobertura de la pobreza entre 2005 y 2014: Qué ha cambiado y qué no en los noticiarios de televisión abierta en Chile", Cuadernos.info, 36, pp. 53-69, DoI: http://dx.doi.org/10.7764/cdi.36.727. 
Falkheimer, J. y E.K. Olsson (2015), “Depoliticizing Terror: The News Framing of the Terrorist Attacks in Norway, 22 July 2011”, Media, War \& Conflict, 8(1), pp. 70-85.

Feenstra, R.A. y A. Casero-Ripollés (2014), "Democracy in the Digital Communication Environment: A Typology Proposal of Political Monitoring Processes”, International Journal of Communication, 8, pp. 2448-2468.

Fox, J.A. (2015), “Social Accountability: What Does the Evidence Really Say?” World Development, 72, pp. 346-361, Dor: https://doi.org/10.1016/j.worlddev.2015.03.011. Garcia, S.M., L.A. Muñoz y A.C. Ripollés (2017), "Usos ciudadanos de Twitter en eventos políticos relevantes. La\# SesiónDeInvestidura de Pedro Sánchez”, Comunicación y Hombre, 13 , pp. 25-49.

García-Perdomo, V. (2017), "Entre paz y odio: Encuadres de la elección presidencial colombiana de 2014 en Twitter”, Cuadernos.info, 41, pp. 57-70, Dor: http://dx.doi.org/ 10.7764/ cdi.41.1241.

Gearhart, S. y D. Dinkel (2016), "Mother Knows Breast: A Content Analysis of Breastfeeding in Television Network News”, Health Communication, 31(7), pp. 884-891, DoI: https://doi.org/10.1080/10410236.2015.1012631.

Gibs, J., H. Shimmel, D. Kaplan y C. Schilling (2009), The Shifting Media Landscape: Integrated Measurement in a Multi-screen World, Nueva York, The Nielsen Company.

Gil de Zúniga, H., L. Molyneux y P. Zheng (2014), "Social Media, Political Expression, and Political Participation: Panel Analysis of Lagged and Concurrent Relationships", Journal of Communication, 64(4), pp. 612-634, Dor: https://doi.org/10.1111/jcom.12103.

Gil de Zúñiga, H., V. García-Perdomo y S.C. McGregor (2015), "What is Second Screening? Exploring Motivations of Second Screen use and Its Effect on Online Political Participation”, Journal of Communication, 65(5), pp. 793-815, Dor: https://doi.org/10.1111/ jcom. 12174.

Gil de Zúñiga, H. y J.H. Liu (2017), "Second Screening Politics in the Social Media Sphere: Advancing Research on Dual Screen Use in Political Communication with Evidence from 20 Countries", Journal of Broadcasting \& Electronic Media, 61(2), pp. 193-219, Dor: https://doi.org/10.1080/08838151.2017.1309420.

Gorkovenko, K. y N. Taylor (2016), "Politics at Home: Second Screen Behaviours and Motivations During TV Debates", Proceedings of the 9 th Nordic Conference on HumanComputer Interaction, octubre, p. 22.

Grundberg, M.D. y S. Lindgren (2015), “Translocal Frame Extensions in a Networked Protest: Situating the\# IdleNoMore Hashtag”, IC Revista Cientifica de Información y Comunicación, 11.

Hampton, M. (2010), “The Fourth Estate Ideal in Journalism History”, en A. Stuart (ed.), The Routledge Companion to News and Journalism, Abingdon, Routledge. 
Hermida, A. (2010), "Twittering the News: The Emergence of Ambient Journalism", Journalism Practice, 4(3), pp. 297-308, DoI: https://doi.org/10.1080/17512781003640703. Holody, K.J. y E.S. Daniel (2017), "Attributes and Frames of the Aurora Shootings: National and Local News Coverage Differences", Journalism Practice, 11(1), pp. 80-100, DoI: https://doi.org/10.1080/17512786.2015.1121786.

Hootsuite (2020), \#Digital 2020: Chile, Nueva York, Hootsuite.

Iyengar, S. (1991), Is Anyone Responsible? How Television Frames Political Issues, Chicago, The University of Chicago Press.

Iyengar, S. y A. Simon (1993), "News Coverage of the Gulf Crisis and Public Opinion: A Study of Agenda-setting, Priming and Framing", Communication Research, 20(3), pp. 365-383.

Jacobson, S. (2013), "Does Audience Participation on Facebook Influence the News Agenda? A Case Study of the Rachel Maddow Show", Journal of Broadcasting \& Electronic Media, 57(3), pp. 338-355, Dor: https://doi.org/10.1080/08838151.2013.816706.

Jha, C.K. y S. Sarangi (2017), “Does Social Media Reduce Corruption?” Information Economics and Policy, 39, pp. 60-71, DoI: https://doi.org/10.1016/j.infoecopol.2017.04.001. Jensen, M. (2016), "The Emergence of Second-screen Gatekeeping”, Digital Journalism, 4(3), pp. 321-338, Dor: https://doi.org/10.1080/21670811.2015.1054408.

Jung, J.Y. y M. Villi (2018), "Newspapers and Cross-Level Communications on Social Media: A Comparative Study of Japan, Korea, and Finland", Digital Journalism, 6(1), pp. 58-75, DOI: 10.1080/21670811.2017.1335605.

Kantar (2017), Rating canales chilenos, Kantar Iворе Media.

Keane, J. (2011), "Monitory Democracy?” en S. Alonso, J. Keane y W. Merkel (eds.), The Future of Representative Democracy, Cambridge, Cambridge University Press, pp. 212235, Dor: $10.1017 / \mathrm{CBO} 9780511770883.010$.

Kepplinger, H.M., S. Geiss y S. Siebert (2012), "Framing Scandals: Cognitive and Emotional Media Effects", Journal of Communication, 62(4), pp. 659-681, Dor: https://doi. org/10.1111/j.1460-2466.2012.01653.x.

Krippendorff, K. (1990), Metodología y análisis de contenido, Barcelona, Paidós.

Larsen, A.G. (2018), "Threatening Criminals and Marginalized Individuals: Frames and News Conventions in Reporting of Radicalization and Violent Extremism", Media, War \& Conflict, 12(3), pp. 299-316, Dor: https://doi.org/10.1177/1750635218769331.

Latinobarómetro (2016), Informe 2015, Buenos Aires, Latinobarómetro.

Latinobarómetro (2017), Informe 2016, Buenos Aires, Latinobarómetro.

Latinobarómetro (2019), Informe 2018, Buenos Aires, Latinobarómetro.

McCombs, M. (2006), Estableciendo la agenda: El impacto de los medios en la opinión pública y en el conocimiento, Barcelona, Paidós. 
McCombs, M. y D. Shaw (1972), "The Agenda Setting Function of Mass Media”, The Public Opinión Quarterly, 36(2), pp. 176-187, DoI: https://doi.org/10.1086/267990.

McCombs, M., D. Shaw y D. Weaver (2014), "New Directions in Agenda-Setting Theory and Research", Mass Communication and Society, 17(6), pp. 781-802, Dor: https://doi. org/10.1080/15205436.2014.964871.

McQuail, D. (2013), Journalism and Society, Thousand Oaks, Sage.

McHale, J.P. (2017), "Media Coverage of Corruption and Scandal in the 2016 Presidential Election: Fantasy Themes of Crooked Hillary and Corrupt Businessman Trump", Corruption, Accountability and Discretion, 29, pp. 107-123.

Meraz, S. (2009), "Is There an Elite Hold? Traditional Media to Social Media Agenda Setting Influence in Blog Networks", Journal of Computer-mediated Communication, 14(3), pp. 682-707, DoI: https://doi.org/10.1111/j.1083-6101.2009.01458.x.

Morales, M.C., N.G. Cabezas, K. Jaime y F. Rendić (2018), "Uso de Twitter en desastres: El terremoto de Iquique”, Interciencia, 43(5), pp. 343-350.

Myrick, J.G., L.H. Major y S.M. Jankowski (2014), "The Sources and Frames Used to Tell Stories about Depression and Anxiety: A Content Analysis of 18 Years of National Television News Coverage", Electronic News, 8(1), pp. 49-63, Dor: https://doi. org/10.1177/1931243114523962.

Neate, T., M. Jones y M. Evans (2017), "Cross-device Media: A Review of Second Screening and Multi-device Television”, Personal and Ubiquitous Computing, 21(2), pp. 391405, DoI: https://doi.org/10.1007/s00779-017-1016-2.

Nölleke, D., C.G. Grimmer y T. Horky (2017), "News Sources and Follow-up Communication: Facets of Complementarity between Sports Journalism and Social Media", Journalism Practice, 11(4), pp. 509-526, DoI: https://doi.org/10.1080/17512786.2015.11 25761.

O’Donnell, G. (1996), “Otra institucionalización”, Política y Gobierno, III(2), pp. 219-244.

O’Donnell, G. (2004), "Notas sobre la democracia en América Latina”, preparado para el Proyecto sobre el Desarrollo de la Democracia en América Latina (Proddal), en PNUD, La democracia en América Latina. Hacia una democracia de ciudadanas y ciudadanos. Anexo: El debate conceptual sobre la democracia (CD ROM), Buenos Aires, Aguilar/Altea/ Taurus/Alfaguara.

Park, C. (2012), "How the Media Frame Political Corruption Episodic and Thematic Frames Stories Found Illinois Newspaper", presentado en el Ethics and Reform Symposium on Illinois Government, Chicago, septiembre.

Peruzzotti, E. y C. Smulovitz (2002), Civil Society, the Media and Internet as Tools for Creating Accountability to Poor and Disadvantaged Groups, United Nations Development Programme-Human Development Report Office. 
Peruzzotti, E. y C. Smulovitz (2006), "Social Accountability: An Introduction”, en E. Peruzzotti y C. Smulovitz (eds.), Enforcing the Rule of Law: Social Accountability in the New Latin American Democracies, Pittsburgh, University of Pittsburgh Press.

Peruzzotti, E. (2019), "Scandals and Social Accountability", en H. Tumber y S. Waisbord (eds.), The Routledge Companion to Media and Scandal, Londres, Routledge.

Przeworski, A. (2006), "Accountability social en América Latina y más allä", en E. Peruzzotti y C. Smulovitz (eds.), Enforcing the Rule of Law: Social Accountability in the New Latin American Democracies, Pittsburgh, University of Pittsburgh Press.

Prabowo, H.Y., R. Hamdani y Z.M. Sanusi (2018), "The New Face of People Power: An Exploratory Study on the Potential of Social Media for Combating Corruption in Indonesia", Australasian Accounting, Business and Finance Journal, 12(3), pp. 19-20, DOI: http://dx.doi.org/10.14453/aabf.v12i3.3.

Rose-Ackerman, S. y B.J. Palifka (2016), Corruption and Government: Causes, Consequences, and Reform, Cambridge, Cambridge University Press.

Rosenstiel, T., J. Sonderman, K. Loker, M. Ivancin y N. Kjarval (2015), "Twitter and the News: How People Use the Social Network to Learn about the World”, disponible en: https://www. americanpressinstitute. org/publications/reports/survey-research/howpeople-use-twitter-news/single-page [fecha de consulta: 12 de agosto de 2021].

Sádaba, T., J. Rodríguez y M. Bartolomé (2012), "Propuesta de sistematización de la teoría del framing para el estudio y praxis de la comunicación política”, Observatorio (OBS*) Journal, 2(6), pp. 109-126.

Scheufele, D. y D. Tewksbury (2007), "Framing, Agenda Setting and Priming: The Evolution of Three Media Effects Models", Journal of Communication, 57(1), pp. 9-20, DOI: https://doi.org/10.1111/j.1460-2466.2006.00326_5.x.

Scheufele, D. y S. Iyengar (2012), "The State of Framing Research: A Call for New Directions", en K. Kenski y K.H. Jamieson (eds.), The Oxford Handbook of Political Communication Theories, Nueva York, Oxford University Press.

Semetko, H. y P. Valkenburg (2000), "Framing European Politics: A Content Analysis of Press and Television News", International Communication Association, 50(2), pp. 93-109, DOI: https://doi.org/10.1111/j.1460-2466.2000.tb02843.x.

Tagle, F. (2017a), "Alcances y obstáculos de la accountability mediática en América Latina tras la Tercera Ola de la democracia", Anagramas-Rumbos y sentidos de la comunicación, 16(31), pp. 135-150.

Tagle, F. (2017b), "Framing y desastres naturales: Encuadre a la prensa escrita chilena", Perspectivas de la Comunicación-ISSN 0718-4867, 10(1), pp. 137-156.

Tagle, F. (2021), "Generic Frames in Corruption Scandals in Chile (2015-2019): Differences and Similarities Between Print and Online Media”, International Journal of Com- 
munication, 15(22), disponible en: <https://ijoc.org/index.php/ijoc/article/view/16852> [fecha de consulta: 12 de agosto de 2021].

Tagle, F. y C. Claro (2018), "Encuadres y uso de fuentes en la vigilancia mediática. Análisis de prensa argentina y chilena: Casos José López y Caval”, Estudios sobre el Mensaje Periodístico, 24(1), p. 413, DoI: https://doi.org/10.5209/ESMP.59958.

Tankard, J. (2001), "The Empirical Approach to the Study of Media Framing”, en S.D. Reese, O.H. Gandy, Jr. y A.E. Grant (eds.), Framing Public Life: Perspectives on Media and Our Understanding of the Social World, Londres, Routledge, pp. 95-106.

Teletrece (2017a), "10 detenidos por fraude en Carabineros: Detenido ex director de Finanzas", 18 de marzo, Santiago de Chile, Canal 13.

Teletrece (2017b), "Fraude en Carabineros suma 15 oficiales", 10 de marzo, Santiago de Chile, Canal 13.

Teletrece (2017c), "Último minuto: Nuevas detenciones en Carabineros", 11 de marzo, Santiago de Chile, Canal 13.

Thompson, J. (2001), El escándalo político: Poder y visibilidad en la era de los medios de comunicación, Barcelona, Paidós.

TI (Transparency International) (2017), Corruption Perceptions Index 2016, Washington, D.C., Transparency International.

TI (Transparency International) (2019), Corruption Perceptions Index 2018, Washington, Transparency International.

Trilling, D. (2015), "Two Different Debates? Investigating the Relationship between a Political Debate on TV and Simultaneous Comments on Twitter", Social Science Computer Review, 33(3), pp. 259-276, DoI: https://doi.org/10.1177/0894439314537886.

Valenzuela, S., T. Correa y H. Gil de Zuniga (2018), “Ties, Likes, and Tweets: Using Strong and Weak Ties to Explain Differences in Protest Participation Across Facebook and Twitter use", Political Communication, 35(1), pp. 117-134, Dor: https://doi.org/10.10 80/10584609.2017.1334726.

Vargo, C.J., E. Basilaia y D.L. Shaw (2015), “Event versus Issue: Twitter Reflections of Major News, a Case Study”, en L. Robinson, S.R. Cotten y J. Schulz (eds.), Communication and Information Technologies Annual: Politics, Participation and Production, 9, Bingley, Emerald Group Publishing, pp. 215-239.

Waisbord, S. (2000), Watchdog Journalism in South America. News, Accountability, and Democracy, Nueva York, Columbia University Press.

Waisbord, S. (2012), "Political Communication in Latin America", en H. Semetko y M. Scammell (eds.), The Sage Handbook of Political Communication, Nueva Delhi, Sage. Weeks, B.E., A. Ardèvol-Abreu y H. Gil de Zúńiga (2017), "Online Influence? Social Media Use, Opinion Leadership, and Political Persuasion”, International Journal of 
Public Opinion Research, 29(2), pp. 214-239, Dor: https://doi.org/10.1093/ijpor/edv050. Weeks, B.E. y R.L. Holbert (2013), "Predicting Dissemination of News Content in Social Media a Focus on Reception, Friending, and Partisanship", Journalism \& Mass Communication Quarterly, 90(2), pp. 212-232, DoI: 10.1177/1077699013482906.

Youyou, W., M. Kosinski y D. Stillwell (2015), "Computer-based Personality Judgments are More Accurate than Those Made by Humans", Proceedings of the National Academy of Sciences, 112(4), pp. 1036-1040, Dor: https://doi.org/10.1073/pnas.1418680112. Zamora, R. y J. Marín Albaladejo (2010), "La representación simbólica del escándalo político: Hacia una tipología de los marcos periodísticos (Frames) utilizados en la narración del escándalo de corrupción política", Razón y Palabra, 73, pp. 1-27.

Francisco Javier Tagle Montt es académico de la Facultad de Comunicación de la Universidad de los Andes, Chile. Doctor en Estudios Latinoamericanos por la Universidad de Salamanca, España.

Juan José Guerrero Cortés es académico de la Facultad de Comunicación de la Universidad de los Andes, Chile. Magíster en Comportamiento del Consumidor, Universidad Adolfo Ibáñez, Chile.

María Paz Vial Cerda es licenciada en Comunicación Social por la Universidad de los Andes, Chile. 